\title{
Efficacy and central mechanism of acupuncture treatment in patients with neck pain: study protocol for a randomized controlled trial
}

Zhen Gao ${ }^{1 \dagger}$, Tao Yin ${ }^{1 \dagger}$, Lei Lan ${ }^{1 \dagger}$, Dehua Li ${ }^{2}$, Ruirui Sun ${ }^{1}$, Guodong Ha ${ }^{1}$, Caili Jiang ${ }^{1}$, Xin Shao ${ }^{3}$, Zhaoxuan He , Laixi $\mathrm{Ji}^{4^{*}}$ and Fang Zeng ${ }^{1 *}$

\begin{abstract}
Background: Acupuncture is effective for reducing the symptoms of neck pain (NP). However, the underlying mechanisms are not fully elucidated. Based on evaluating the efficacy of two acupuncture prescriptions for treating $N P$, this study aims to investigate the potential central mechanism of acupuncture treatment for NP by functional magnetic resonance imaging (fMRI).

Methods: This is a randomized controlled trial; 86 patients will be randomly assigned into two acupuncture treatment groups at a ratio of 1:1. The whole study period includes 2 weeks baseline, 2 weeks treatments, and 12 weeks follow-up ( 4 and 12 weeks after treatment). The pain severity, the neck disability index, the cervical range of motion, and the pressure pain threshold, etc., will be used to evaluate the clinical efficacy of two acupuncture prescriptions for NP treatment. The MRI scans will be performed to detect cerebral activity changes of 20 patients in each group. The clinical data and MRI data will be analyzed, respectively. Pearson correlation coefficient will be used to evaluate the association between changes of cerebral activity features and improvement of clinical symptoms.
\end{abstract}

Discussion: The results will provide further evidence for the clinical application of acupuncture in the treatment of NP. Trial registration: Chinese Clinical Trial Registry ChiCTR2000040930. Registered on 16 December 2020.

Keywords: Neck pain, Acupuncture, Central mechanism, Functional magnetic resonance imaging, Protocol

\footnotetext{
*Correspondence: tyjilaixi@126.com; zeng_fang@126.com

${ }^{\dagger}$ Zhen Gao, Tao Yin, and Lei Lan contributed equally to this work and are cofirst authors.

${ }^{4}$ Graduate Faculty, Shanxi University of Traditional Chinese Medicine, 121\# University Street, Jinzhong 030619, Shanxi, China

'Brain Research Center, Acupuncture and Tuina School/The 3rd Teaching Hospital, Chengdu University of Traditional Chinese Medicine, 37\# Shierqiao Road, Chengdu 610075, Sichuan, China

Full list of author information is available at the end of the article
}

(c) The Author(s). 2021 Open Access This article is licensed under a Creative Commons Attribution 4.0 International License, which permits use, sharing, adaptation, distribution and reproduction in any medium or format, as long as you give appropriate credit to the original author(s) and the source, provide a link to the Creative Commons licence, and indicate if changes were made. The images or other third party material in this article are included in the article's Creative Commons licence, unless indicated otherwise in a credit line to the material. If material is not included in the article's Creative Commons licence and your intended use is not permitted by statutory regulation or exceeds the permitted use, you will need to obtain permission directly from the copyright holder. To view a copy of this licence, visit http://creativecommons.org/licenses/by/4.0/. The Creative Commons Public Domain Dedication waiver (http://creativecommons.org/publicdomain/zero/1.0/) applies to the data made available in this article, unless otherwise stated in a credit line to the data. 


\section{Background}

Neck pain (NP), as a common musculoskeletal disorder, has become an important healthcare and social problem due to its high prevalence and heavy economic burden [1]. It is estimated that more than $30 \%$ of the population suffer from NP each year [2], and its lifetime prevalence in adults ranges from 14.2 to $71 \%$ [3]. Due to the increasing prevalence of NP, the health care costs of NP have rapidly increased [4-6].

In the United States, the cumulative annual treatment costs for lower back pain and NP is about $\$ 87.6$ billion [7]. The treatment of NP has been challenging, because its etiology is complex and the symptoms are easy to recur. Although medications (e.g., muscle relaxants and nonsteroidal anti-inflammatory drugs) can effectively alleviate acute pain, their side effects, including hepatotoxicity, etc., cannot be ignored [8]. Therefore, more patients tend to purchase non-drug therapies to relieve symptoms.

Acupuncture has been used to relieve pain for thousands of years in China. As early as 1997, the National Institutes of Health (NIH) recommended acupuncture treatment for pain disorders. Evidence from recent large-scale clinical studies has confirmed that acupuncture can effectively improve NP symptoms [9-14]. However, the underlying mechanisms of acupuncture for NP have not been fully elucidated.

Advances in neuroimaging techniques have enhanced studies into the central mechanisms of acupuncture as an analgesic in vivo. In the last two decades, many neuroimaging studies have been performed to elucidate the mechanisms of acupuncture analgesia, and achieved gratifying results [15-18]. For example, an fMRI study on migraines found that acupuncture was effective in improving abnormally reduced functional activity in the rostral ventromedial medulla/trigeminocervical complex area of patients [19]. Recently, significant alterations in the brain structure and function of NP patients have been reported by neuroimaging studies. Compared to healthy subjects, NP patients shown decreased functional activity in the left sensorimotor cortex and right temporoparietal junction [20], as well as increased functional connectivity between the right dorsolateral prefrontal cortex and the right anterior insula, which are associated with pain intensity [21]. Based on these findings, neuroimaging techniques appear to be useful tools for investigating the central mechanisms of acupuncture therapy for NP. Therefore, by MRI, this study aims to (1) evaluate the therapeutic effects of different acupoints prescription on NP, (2) investigate the changes in cerebral activity elicited by acupuncture treatment, and (3) analyze the possible correlations between the improvement of clinical symptoms and changes in brain activity, so as to explore the potential central mechanism of acupuncture for NP.

\section{Methods and design \\ Study design}

This is a randomized controlled trial. This protocol is reported in accordance with the Standard Protocol Items: Recommendations for Intervention Trials (SPIRIT) guidelines (supplementary file 1) and follows the principles of the Consolidated Standards of Reporting Trials (CONSORT) and the Standards for Reporting Interventions in Clinical Trials of Acupuncture (STRICTA) [22, 23]. The protocol has been approved by the Institutional Review Board of the Hospital of Chengdu University of Traditional Chinese Medicine (approved number: 2020KL-007) and registered at Chinese Clinical Trial Registry (registration number: ChiCTR2000040930, protocol version number: V1.0).

This study period last for 16 weeks, which included a 2-week baseline period, a 2-week intervention period, and a 12-week following period. A total of 86 patients with NP will be randomly assigned to the two acupuncture groups with a 1:1 ratio. Twenty patients in each group will be randomly selected to undertake MRI scans. Clinical outcome evaluation and MRI scans will be conducted at baseline and after treatment. The study procedure is outlined in Fig. 1 and Table 1.

\section{Participants \\ Recruitment strategy}

Patients will be recruited at the outpatient of the Hospital of Chengdu University of Traditional Chinese Medicine (TCM) and Sichuan Integrated Medicine Hospital. In addition, patients will also be recruited from the community and campus of Chengdu University of TCM through advertising (posting notices, distributing leaflets, web publishing). Patients who agree to participate in this study will be diagnosed by an orthopedic specialist. The eligible patients will be screened by the research assistant based on the inclusion and exclusion criteria.

\section{Inclusion criteria}

Participants who match the inclusion criteria will be included: (1) patients with NP as the main complaint and a visual analog scale (VAS) score of pain severity exceeding 4 points but less than 10 points (range $0-10$ points), (2) for a duration of $\leq 3$ months, (3) aged 18-60 years, and (4) agreeing to sign informed consent.

\section{Exclusion criteria}

Participants with any of the following conditions will be excluded: (1) they are pregnant or lactating women; (2) they have any organic or metabolic diseases of the digestive, hematopoietic, endocrine, or immune systems, or other severe primary diseases; (3) they have 


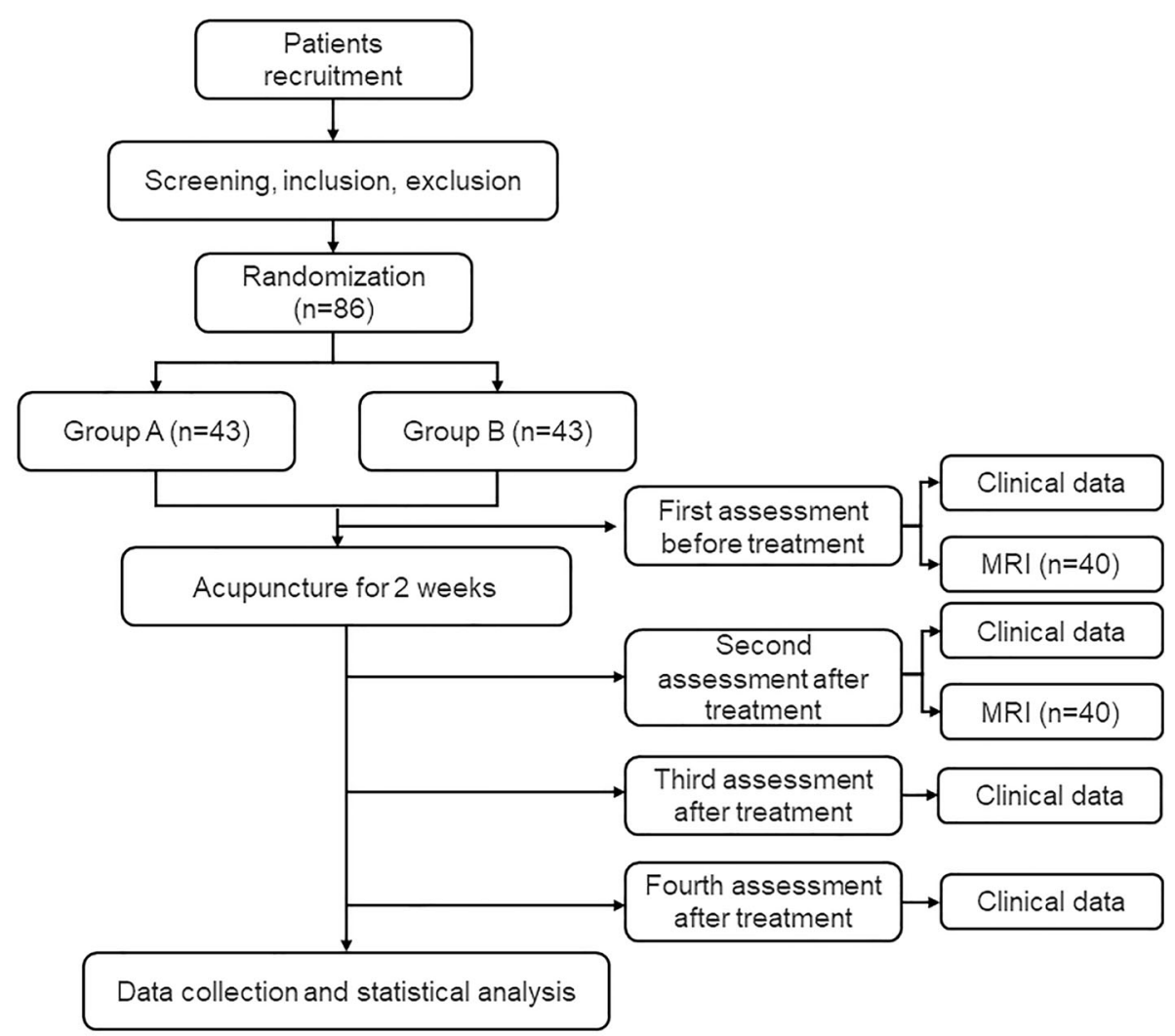

Fig. 1 Flowchart of the procedure through the trial. The randomized controlled trial will enroll 86 eligible patients and divide them randomly into two groups at a 1:1 ratio. Twenty patients from each group will be randomly selected for MRI data collection. During the 2-week treatment, the patient will receive 6 acupuncture treatments. Clinical assessment will be performed at 4 time points: baseline, end of treatment, and 6 and 14 weeks after completion. The MRI scans will be performed at baseline and after treatment. Abbreviations: MRI magnetic resonance imaging

fibromyalgia and other central sensitization pain syndromes; (4) they have local skin damage or severe skin diseases that affect acupuncture manipulation; (5) they combine with mental or neurological diseases; (6) they have received acupuncture for NP within 1 month; (7) they have MRI contraindications, such as heart pacemaker, metallic foreign bodies, or severe claustrophobia, etc.; or (8) they are participating in other clinical trials.

\section{Sample size}

This study aims to compare the difference of the clinical efficacy of two acupuncture prescriptions (group A and group B). According to the results of the pilot study, the average reduction of VAS score in group A was 2.86 \pm 0.99 , and the average reduction of VAS score in group B was 2.14 \pm 0.99 . According to the formula, $n=\frac{2 \sigma^{2}}{(\mu 1-\mu 2)^{2}}$ $\times\left(\mu_{\alpha / 2}+\mu_{\beta}\right)^{2}$, with $\alpha=0.05$ (both sides) and $1-\beta=$ 0.80 [24]. The sample size of each group was calculated to be 34 , considering a $20 \%$ dropout rate; the final sample size was set to 43 per group, making the total of 86 in this study.
There is no widely accepted method for sample size calculation in neuroimaging study. Referring to the previous study [25], 15 participants in each group are the smallest size in neuroimaging studies. Considering a $20 \%$ dropout rate and the unavailability of data due to various reasons, this study will randomly select 20 patients from each group for MRI scans.

\section{Randomization}

Randomization will be carried out in two steps: (1) an independent, blinded statistician will generate a random allocation sequence using SPSS 26.0 (IBM, Chicago, IL, USA). The 86 patients will be randomly assigned into group A and group B at a ratio of 1:1; (2) 20 patients in each group will be randomly selected for MRI according to another random sequence. Allocation concealment will be achieved by sequentially numbered, opaque, sealed envelopes.

\section{Blinding}

In this study, the patients, outcome assessors, and statistical analysts will be blinded. The patients will be treated in separate rooms to reduce communication. However, 
Table 1 Study schedule for data collection

\begin{tabular}{|c|c|c|c|c|c|c|}
\hline \multirow{2}{*}{$\begin{array}{l}\text { Period } \\
\text { Assessment }\end{array}$} & \multicolumn{2}{|c|}{ Baseline } & \multicolumn{2}{|l|}{ Treatment } & \multicolumn{2}{|c|}{ Follow-up } \\
\hline & & 1 & & 2 & 3 & 4 \\
\hline Time (week) & 0 & 2 & 3 & & 8 & 16 \\
\hline \multicolumn{7}{|l|}{ Pick-up information } \\
\hline Inclusion/exclusion criteria & $\sqrt{ }$ & & & & & \\
\hline Informed consent & $\sqrt{ }$ & & & & & \\
\hline Vital signs & $\sqrt{ }$ & & & & & \\
\hline Medical history & $\sqrt{ }$ & & & & & \\
\hline Physical examination & $\sqrt{ }$ & & & $\sqrt{ }$ & & \\
\hline
\end{tabular}

Acupuncture intervention

$\begin{array}{lllllll}\text { Group A }(n=43) & \sqrt{ } & \sqrt{ } & \sqrt{ } & \sqrt{ } & \sqrt{ } & \sqrt{ } \\ \text { Group B }(n=43) & \sqrt{ } & \sqrt{ } & \sqrt{ } & \sqrt{ } & \sqrt{ } & \sqrt{ }\end{array}$

Clinical assessment

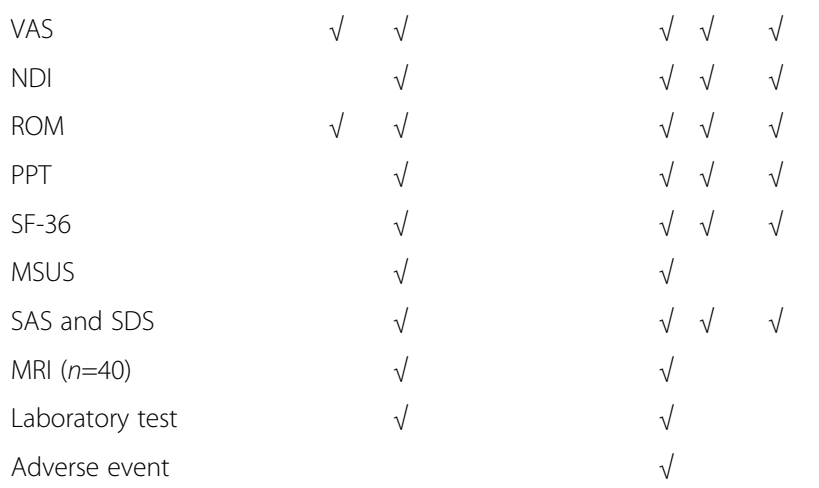

This is a randomized controlled trial of neuroimaging including a 2-week baseline period, a 2-week treatment period, and a 12-week follow-up period. At baseline, patients will be screened based on inclusion and exclusion criteria; eligible individuals will then sign informed consent and undergo a physical examination. After being randomized into 2 groups, 6 acupuncture treatments will be performed for 2 weeks. Clinical data will be evaluated at baseline, after treatment ends, and at 4 and 12 weeks after completion; MRI scans will be performed at baseline and after treatment. After the treatment, laboratory tests will be performed, including routine blood test, C-reactive protein, and erythrocyte sedimentation tests. Adverse events will be recorded in the CRF at any time during treatment. Abbreviations:MRI magnetic resonance imaging, VAS visual analogue scale, NDI neck disability index, ROM range of motion, PPT pressure pain thresholds, MSUS musculoskeletal ultrasound, SF-36 ShortForm 36-Item Health Survey, SAS Self-rating Anxiety Scale, SDS Self-rating Depression Scale, CRF case report form

due to the particularity of acupuncture manipulation, blinding operator cannot be achieved.

\section{Interventions}

Patients in group A will receive acupuncture at three acupoints (Lieque (LU7), Chize (LU5), and neck tenderness point on the affected side) (Fig. 2A). The acupoints of group B (control group) will receive acupuncture at three acupoints (Shaohai (HT3), Lingdao (HT4), and neck tenderness point on the affected side) (Fig. 2B). Tenderness points will be detected using a PX25 handheld pressure pain tester (Wagner FPX ${ }^{\text {su }} 25$ ).

The acupuncture manipulations are as follows: disposable sterile filiform needles $(0.30 \times 40 \mathrm{~mm}$, Huatuo
Medical Instrument Co., Ltd., China) will be inserted into acupoints at a depth of $20-30 \mathrm{~mm}$ after skin disinfection, and the Deqi sensation (a sensation of acid distension or numbness, or other acupuncture sensation) will be achieved [26]. Then, the HANS-200A acupoint nerve stimulator (Nanjing Jisheng Medical Technology Company, Nanjing city, China) will connect LU7 and LU5 of group A or HT3 and HT4 of group B respectively in the two groups for $30 \mathrm{~min}$ with dilatational waves $(2-100 \mathrm{~Hz}, 1 \mathrm{~mA})$. Patients will receive a total of 6 sessions of acupuncture in two weeks, with 3 sessions per week.

Acupuncture treatment will be performed by acupuncturists who have held a practitioner license for more than 3 years.

\section{Concomitant medications}

Patients will be instructed not to take any other analgesic medications for NP during this study. In cases of severe NP, ibuprofen (300 mg per capsule with sustained release) will be allowed as rescue medication and should be recorded on the case report form (CRF). Patients are also asked to report to the researchers if they take any concomitant medications during the study, and to record the name, dose, and date of the medications used.

\section{Outcomes}

The measurements will be conducted by independent assessors who have been trained before the study. The primary outcome is the change of VAS scores at baseline and after the end of treatment. VAS scores range from 0 to 10 , with 0 indicating no pain and 10 indicating pain is unbearable. The secondary outcome measurements include neck disability index (NDI), Short-Form 36-Item Health Survey (SF-36), Self-rating Anxiety Scale (SAS), Self-rating Depression Scale (SDS), range of motion (ROM), and pressure pain threshold (PPT) will be evaluated at baseline, after treatment, and at 4 and 12 weeks after the end of treatment. In addition, we will perform musculoskeletal ultrasound (MSUS) before and after treatment. Among them, NDI is the most widely used clinical tool for selfassessment of disability caused by NP [27]. The SAS and SDS are the commonly used psychometric tools to measure the severity of anxiety and depression [28, 29]. SF-36 is a general health-based survey of quality of life, which can be self-administered by the patient with reliability [30]. ROM is one of the quantitative outcomes assessing the extent and degree of joint motor impairment. The changes of the tenderness threshold can quantitatively reflect the improvement of the pain severity [31]. Meanwhile, MSUS is an important method for clinical diagnosis of rehabilitation medicine, tracking disease progression and assessing curative effect [32]. 

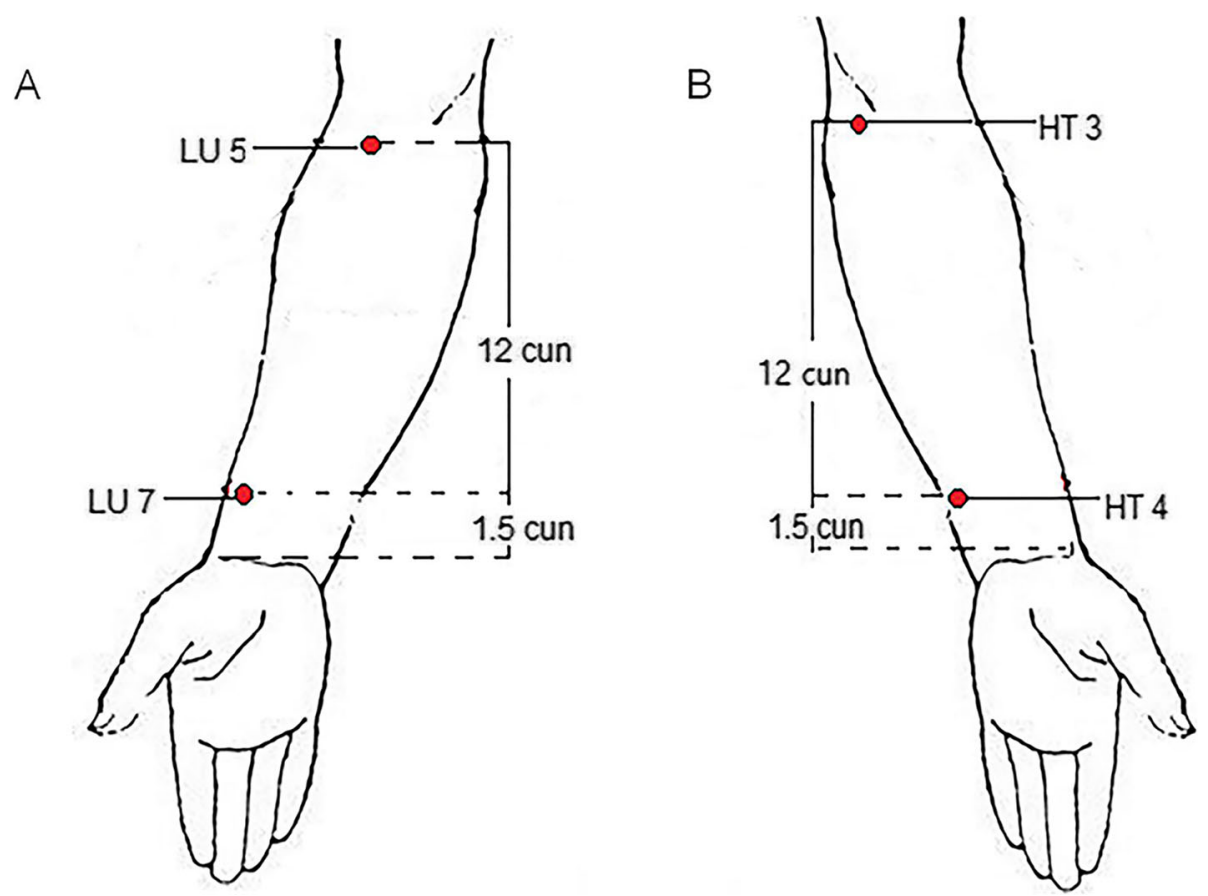

Fig. 2 Locations of acupoints. A Locations of acupoints (group A). Lieque (LU 7), on the radial side of the forearm, above the styloid process of the radius and 1.5 cun above the horizontal stripes of the wrist. Chize (LU 5), in the transverse elbow, the radial side of the biceps tendon is sunken. B Locations of acupoints (group B). Lingdao (HT 4), in the anterior area of the forearm, the radial side of the ulnar carpi flexor tendon is 1.5 cun above the transverse stripes of the carpal palm. Shaohai (HT 3), at the midpoint of the line between the medial end of the transverse line of the elbow and the medial epicondyle of the humerus

\section{Patient safety}

Adverse events during the study period will be reported in the CRF. The record should include the time, reason, clinical symptoms, signs of the adverse event, and the corresponding emergency treatment plan, as well as abnormal laboratory tests with clinical significance. In addition, all events will be evaluated for their relevance to the intervention and severity, according to the clinician.

\section{MRI data acquisition}

The resting-state fMRI (RS-fMRI) will be performed at the baseline and the end of treatment, respectively. Patients will be asked to do not drink tea, coffee, or alcohol, avoid strenuous exercise, and ensure adequate sleep before the scan.

Patients will undergo RS-fMRI scan with a 3.0T MR scanners (Siemens 3T Tim trio, Erlangen, Germany) at the West China Hospital of Sichuan University. Patients will be asked to stay awake and to keep their heads still during the scan, with their eyes closed and ears plugged. Scans will be performed with the following procedures: localizer, three-dimensional T1-weighted imaging (3DT1WI), blood oxygenation level-dependent fMRI (BOLD-fMRI) and diffusion tensor imaging (DTI) sequence. The 3D-T1WI scanning parameters will be as follows: repetition time $(\mathrm{TR})=6.008 \mathrm{~ms}$, echo time $(\mathrm{TE})$ $=1.7 \mathrm{~ms}$, data matrix $=256 \times 256$, field of view $(\mathrm{FOV})=$ $256 \times 256 \mathrm{~mm}^{2}$. The BOLD-fMRI scanning parameters will be as follows: 31 contiguous slices with a slice thickness of $5 \mathrm{~mm}, \mathrm{TR}=2000 \mathrm{~ms}, \mathrm{TE}=30 \mathrm{~ms}, \mathrm{FOV}=240 \times$ $240 \mathrm{~mm}^{2}$, Matrix $=64 \times 64$, flip angle $(\mathrm{FA})=90^{\circ}$, total volumes $=240$. The DTI data will be acquired using a single-shot echo planar image sequence with the following parameters: FOV $=256 \times 256 \mathrm{~mm}^{2}$, TR $=8500 \mathrm{~ms}$, matrix $=128 \times 128$, slice thickness $=2 \mathrm{~mm}$ with no gap. Two diffusion-weighted sequences were acquired using gradient values $\mathrm{b}=1000 \mathrm{~s} / \mathrm{mm}^{2}$ and $\mathrm{b}=0 \mathrm{~s} / \mathrm{mm}^{2}$ with the diffusion-sensitizing gradients applied in 64 noncollinear directions.

\section{Data management}

An electronic entry database will be established based on the CRFs project, and the outcome assessors will fill in relevant information in a timely and accurate manner according to the requirements of CRFs. Only outcome assessors have access to the CRFs and perform double-data entry. The Evidence-based Medicine Center of the Hospital of Chengdu University of TCM will be responsible for monitoring the study and data every 3 months. 


\section{Data analysis}

\section{Clinical data analysis}

The comprehensive effectiveness analysis will use the per protocol set (PPS) and the full analysis set (FAS). The FAS population will be used as the primary population for all efficacy analyses. The safety set (SS) included all subjects who received at least one treatment after randomization. When the results of PPS analysis and FAS analysis are inconsistent, PPS and FAS are discussed separately to find out the reasons for the inconsistency. Missing data will be handled using the lastobservation-carried-forward (LOCF) method.

Statistical analysis will be conducted using SPSS 26.0 (IBM, Chicago, IL, USA) statistical software. $P<0.05$ (two-sided) is considered statistically significant. Quantitative data are described with mean \pm standard deviation (SD). Qualitative data are described with frequency and percentage $(N, \%)$. Student's $t$-test and chi-square test will be used to compare the differences between groups at baseline. The assessment of the difference between the two groups will use repeated measures analysis of covariance (ANCOVA), taking into account the baseline values of age, sex and disease duration as covariates. Clinical data on skewed distribution will be compared using a non-parametric test.

\section{BOLD-fMRI data analysis}

The fMRI data preprocessing and comparison will be performed using SPM12 software (http://www.fil.ion.ucl. ac.uk/spm/) based on MATLAB 2018a (Mathworks, Inc., USA). The steps including: format conversion, slice timing correction, head motion correction, spatial standardization, linear trend removal, spatial smoothing, and band-pass filtering. After pre-processing, the amplitude of low frequency fluctuation and regional homogeneity will be calculated to reflect the local cerebral functional activity, and the seeds-based functional connection analysis will be performed to reflect the functional integration of the brain. ANOVA will be used for comparisons between the two groups, with multiple comparison corrections.

Furthermore, the Pearson correlation analysis will be conducted to assess the associations between the changes of fMRI and the improvement of clinical symptoms.

\section{Discussion}

To the best of our knowledge, this is the first randomized controlled study to investigate the clinical efficacy and underlying mechanisms of different acupuncture prescriptions for NP. The results of this study are beneficial for the prescription selection of acupuncture for treating NP and elucidate on the mechanisms of acupuncture for NP.

\section{Selection of acupoint prescription}

Many clinical studies have been performed on acupuncture for NP, and its efficacy has been identified. In clinical practice, the combination of local and distal points is a basic principle of acupoint prescriptions, but the number of prescriptions and acupoints involved is enormous. Therefore, in this study, two different prescriptions will be selected to compare their efficacy and possible mechanisms. Neck tenderness point will be selected as the basic acupoint, which is the most commonly used local acupoint in NP treatment. LU7 and LU5, located in the forearm, will be selected as the distal points in group A. These two points are the effective and common acupoints for neck diseases based on the traditional Chinese medicine theories and clinical practice [33-35]. HT4 and HT3, also located in the forearm, will be selected as the distal points in group B. These two acupoints are located on the same nerve segment as those in group A. This is a commonly available method for determining a control group in acupuncture studies and has been proven to be effective in blinding patients in many studies [36, 37].

\section{The combination of subjective and objective outcome measures}

Pain is an unpleasant sensory and emotional perception that is associated with actual or underlying tissue pathology [38]. Human behavior, psychological, and social factors play a crucial role in the long-term maintenance of pain [39]. Therefore, the multidimensional nature of pain requires that the measurement of NP should include both subjective outcomes and objective outcomes. In this study, subjective outcomes including VAS, NDI, SAS, and SDS will be used to assess pain perception, dysfunction, and emotional state of the patients. Objective outcomes such as tenderness threshold, musculoskeletal ultrasound, and ROM will be used to evaluate the symptom improvement in the NP patients. The combination of subjective and objective outcomes can be more complementary to assess the efficacy from multiple perspectives and reduce the occurrence of potential bias, so as to improve the quality of clinical evidence.

\section{Application of neuroimaging technology}

Previous neuroimaging studies have demonstrated that patients with NP exhibit abnormalities in brain activity and structure. For example, Chen et al. [40] found increased spontaneous brain activity in the supplementary motor area of NP patients and subsequently suggested a correlation between abnormality of the salience network with the disease. Another single fMRI-based case control study associated NP with activation and/or recall of pain memory [41]. Elsewhere, a voxel-based morphometry study performed on NP patients revealed the loss of 
bilateral clusters of gray matter in the sensorimotor cortex and pulvinar nucleus with NP pathophysiology [42].

Moreover, it has been reported that central integration is the key determinant of the effectiveness of acupuncture [43]. With previous studies, performed on individuals with shoulder pain [44], fibromyalgia [45], and low back pain [46], as well as knee osteoarthritis [15] indicating that regulation of brain activity might be an important factor influencing acupuncture's modulating effects on pain. For example, acupuncture has been shown to effectively reduce pain by modulating brain regions related to both sensory-discriminative and emotional aspects [47]. Such regions were found to be important for the therapeutic effects of acupuncture on NP [40]. Based on these evidences, we hypothesize that acupuncture can effectively treat NP by modulating alteration of brain activity.

This trial is the first study to use fMRI to explore the central mechanisms of different acupuncture prescriptions in the treatment of NP. The results of this trial will help to provide visualization evidence for the clinical application of acupuncture for NP treatment.

\section{Trial status}

The trial is currently in the recruitment phase and the study is expected to end in December 2021.

\section{Abbreviations}

3D-T1Wl: Three-dimensional T1-weighted imaging; ANCOVA: Analysis of covariance; BOLD-fMRI: Blood oxygenation level-dependent fMRI; RSfMRI: Resting state functional magnetic resonance imaging; CRF: Case report form; DTI: Diffusion tensor imaging; FA: Flip angle; FAS: Full analysis set; FDR: False discovery rate; FOV: Field of view; GLM: General linear model; HbO: Oxygenated hemoglobin; HbR: Deoxygenated hemoglobin; LOCF: Last observation carry-forward; MRI: Magnetic resonance imaging: MSUS: Musculoskeletal ultrasound; NDI: Neck disability index; NP: Neck pain; $\mathrm{NIH}$ : National Institutes of Health; PPS: Per protocol set; PPT: Pressure pain threshold; ROM: Range of motion; SD: Standard deviation; SF-36: Short-form 36-item Health Survey; SAS: Self-Rating Anxiety Scale; SDS: Self-Rating Depression; SS: Safety set; TCM: Traditional Chinese medicine; TE: Echo time: TR: Repetition time; VAS: Visual analogue scale

\section{Supplementary Information}

The online version contains supplementary material available at https://doi. org/10.1186/s13063-021-05507-y.

Additional file 1. SPIRIT 2013 Checklist: Recommended items to address in a clinical trial protocol and related documents.

\section{Acknowledgements}

We thank Haifa Qiao and Gang Lu for the help of participant enrollment.

\section{Authors' contributions}

$F Z$ and $L J$ are responsible for this study. FZ, LJ, ZG, TY, and $L L$ conceived and designed the study. ZG, TY, and LL participated in drafting the trial protocol and preparing the manuscript. SR, LD, HG, JC, and $\mathrm{HZ}$ participated in the data collection and were in charge of recruitment and treatment of patients. All authors read and approved the final manuscript.

\section{Funding}

The study is financially supported by the National Key Research and Development Program in China (2018YFC1704606).
Availability of data and materials

Not applicable.

\section{Declarations}

\section{Ethics and dissemination}

Ethical approval of this study has been approved by the Ethics Committee of the Hospital of Chengdu University of Traditional Chinese Medicine (No. 2020KL-007). The outcomes of the trial will be disseminated through peerreviewed publications.

\section{Ethics approval and consent to participate}

This trial has been approved by the Institutional Review Boards and Ethics Committees of the First Teaching Hospital of CDUTCM (Approved number: 2020KL-007) and has been registered in the Chinese Clinical Trial Registry (ID: ChiCTR2000040930). Only patients who have signed the informed consent form will be included.

Consent for publication

Not applicable.

\section{Competing interests}

The authors declare that they have no competing interests.

\section{Author details}

${ }^{1}$ Brain Research Center, Acupuncture and Tuina School/The 3rd Teaching Hospital, Chengdu University of Traditional Chinese Medicine, 37\# Shierqiao Road, Chengdu 610075, Sichuan, China. ${ }^{2}$ Hospital of Chengdu University of Traditional Chinese Medicine, Chengdu 610072, Sichuan, China. ${ }^{3}$ Sichuan Integrated Medicine Hospital, Chengdu 610041, Sichuan, China. ${ }^{4}$ Graduate Faculty, Shanxi University of Traditional Chinese Medicine, 121\# University Street, Jinzhong 030619, Shanxi, China.

Received: 29 March 2021 Accepted: 3 August 2021

Published online: 14 August 2021

\section{References}

1. Safiri S, Kolahi AA, Hoy D, Buchbinder R, Mansournia MA, Bettampadi D, et al. Global, regional, and national burden of neck pain in the general population, 1990-2017: systematic analysis of the Global Burden of Disease Study 2017. BMJ (Clinical research ed). 2020;368:m791.

2. Cohen SP. Epidemiology, diagnosis, and treatment of neck pain. Mayo Clinic proceedings. 2015;90(2):284-99. https://doi.org/10.1016/j.mayocp.2014.09. 008.

3. Fejer R, Kyvik KO, Hartvigsen J. The prevalence of neck pain in the world population: a systematic critical review of the literature. European spine journal : official publication of the European Spine Society, the European Spinal Deformity Society, and the European Section of the Cervical Spine Research Society. 2006;15(6):834-48. https://doi.org/10.1007/s00586-0040864-4.

4. Hurwitz EL, Randhawa K, Yu H, Côté P, Haldeman S. The Global Spine Care Initiative: a summary of the global burden of low back and neck pain studies. European spine journal : official publication of the European Spine Society, the European Spinal Deformity Society, and the European Section of the Cervical Spine Research Society. 2018;27(Suppl 6):796-801, DOl: https://doi.org/10.1007/s00586-017-5432-9.

5. Hoy D, March L, Woolf A, Blyth F, Brooks P, Smith E, et al. The global burden of neck pain: estimates from the global burden of disease 2010 study. Annals of the rheumatic diseases. 2014;73(7):1309-15. https://doi.org/10.113 6/annrheumdis-2013-204431

6. Basson CA, Olivier B, Rushton A. Neck pain in South Africa: an overview of the prevalence, assessment and management for the contemporary clinician. The South African journal of physiotherapy. 2019;75(1):1332. https://doi.org/10.4102/sajp.v75i1.1332.

7. Dieleman JL, Baral R, Birger M, Bui AL, Bulchis A, Chapin A, et al. US spending on personal health care and public health, 1996-2013. Jama. 2016; 316(24):2627-46. https://doi.org/10.1001/jama.2016.16885.

8. Cohen SP, Hooten WM. Advances in the diagnosis and management of neck pain. BMJ (Clinical research ed). 2017;358:j3221.

9. Irnich D, Behrens N, Molzen H, König A Gleditsch J Krauss M, et al. Randomised trial of acupuncture compared with conventional massage and 
"sham" laser acupuncture for treatment of chronic neck pain. BMJ (Clinical research ed). 2001;322(7302):1574-8. https://doi.org/10.1136/bmj.322.7302.1 574.

10. MacPherson $\mathrm{H}$, Tilbrook $\mathrm{H}$, Richmond $\mathrm{S}$, Woodman J, Ballard $\mathrm{K}$, Atkin $\mathrm{K}$, et al. Alexander technique lessons or acupuncture sessions for persons with chronic neck pain: a randomized trial. Ann Internal Med. 2015;163(9):653-62. https://doi.org/10.7326/M15-0667.

11. Wong JJ, Shearer HM, Mior S, Jacobs C, Côté P, Randhawa K, et al. Are manual therapies, passive physical modalities, or acupuncture effective for the management of patients with whiplash-associated disorders or neck pain and associated disorders? An update of the Bone and Joint Decade Task Force on Neck Pain and Its Associated Disorders by the OPTIMa collaboration. The spine journal : official journal of the North American Spine Society. 2016;16(12):1598-630. https://doi.org/10.1016/j.spinee.2015.08 024.

12. Yuan QL, Guo TM, Liu L, Sun F, Zhang YG. Traditional Chinese medicine for neck pain and low back pain: a systematic review and metaanalysis. PloS one. 2015;10(2):e0117146. https://doi.org/10.1371/journal. pone.0117146.

13. Chen L, Li M, Fan L, Zhu X, Liu J, Li H, et al. Optimized acupuncture treatment (acupuncture and intradermal needling) for cervical spondylosisrelated neck pain: a multicenter randomized controlled trial. Pain. 2021; 162(3):728-39. https://doi.org/10.1097/j.pain.0000000000002071.

14. Ho LF, Lin ZX, Leung AWN, Chen L, Zhang H, Ng BFL, et al. Efficacy of abdominal acupuncture for neck pain: a randomized controlled trial. PloS one. 2017;12(7):e0181360. https://doi.org/10.1371/journal.pone.01813 60.

15. Kong J, Wang Z, Leiser J, Minicucci D, Edwards R, Kirsch I, et al. Enhancing treatment of osteoarthritis knee pain by boosting expectancy: a functional neuroimaging study. Neurolmage Clinical. 2018;18:325-34. https://doi.org/1 0.1016/j.nicl.2018.01.021.

16. Cao J, Tu Y, Orr SP, Lang C, Park J, Vangel M, et al. Analgesic effects evoked by real and imagined acupuncture: a neuroimaging study. Cerebral cortex (New York, NY: 1991). 2019:29(8):3220-31.

17. Kong J, Kaptchuk TJ, Polich G, Kirsch I, Vangel M, Zyloney C, et al. Expectancy and treatment interactions: a dissociation between acupuncture analgesia and expectancy evoked placebo analgesia. Neurolmage. 2009; 45(3):940-9. https://doi.org/10.1016/j.neuroimage.2008.12.025.

18. Kong J, Kaptchuk TJ, Polich G, Kirsch I, Vangel M, Zyloney C, et al. An fMRI study on the interaction and dissociation between expectation of pain relief and acupuncture treatment. Neurolmage. 2009;47(3):1066-76. https://doi. org/10.1016/j.neuroimage.2009.05.087.

19. Li Z, Zeng F, Yin T, Lan L, Makris N, Jorgenson K, et al. Acupuncture modulates the abnormal brainstem activity in migraine without aura patients. Neurolmage Clinical. 2017;15:367-75. https://doi.org/10.1016/..nicl.2 017.05.013.

20. Chen J, Wang Z, Tu Y, Liu X, Jorgenson K, Ye G, et al. Regional homogeneity and multivariate pattern analysis of cervical spondylosis neck pain and the modulation effect of treatment. Front Neuroscience. 2018;12:900. https://doi. org/10.3389/fnins.2018.00900

21. Ihara N, Wakaizumi K, Nishimura D, Kato J, Yamada T, Suzuki T, et al. Aberrant resting-state functional connectivity of the dorsolateral prefrontal cortex to the anterior insula and its association with fear avoidance belief in chronic neck pain patients. PloS one. 2019;14(8):e0221023. https://doi.org/1 0.1371/journal.pone.0221023.

22. Schulz KF, Altman DG, Moher D. CONSORT 2010 statement: updated guidelines for reporting parallel group randomised trials. Int J Surgery (London, England). 2011;9(8):672-7.

23. MacPherson $H$, Altman DG, Hammerschlag R, Youping L, Taixiang W, White A, et al. Revised STandards for Reporting Interventions in Clinical Trials of Acupuncture (STRICTA): extending the CONSORT statement. PLoS medicine. 2010;7(6):e1000261. https://doi.org/10.1371/journal.pmed.1000261.

24. Chow S, Shao J, Wang H. Sample size calculations in clinical research. 2nd ed. Boca Raton: Chapman and Hall/CRC; 2008.

25. Hayasaka S, Peiffer AM, Hugenschmidt CE, Laurienti PJ. Power and sample size calculation for neuroimaging studies by non-central random field theory. Neurolmage. 2007;37(3):721-30. https://doi.org/10.1016/j.neuroima ge.2007.06.009.

26. Yuan HW, Ma LX, Qi DD, Zhang P, Li CH, Zhu J. The historical development of deqi concept from classics of traditional chinese medicine to modern research: exploitation of the connotation of deqi in chinese medicine.
Evidence-based complementary and alternative medicine : eCAM. 2013; 2013:639302.

27. Blanpied PR, Gross AR, Elliott JM, Devaney LL, Clewley D, Walton DM, et al. Neck Pain: Revision 2017. J Orthopaedic Sports Physical Therapy. 2017;47(7): A1-a83. https://doi.org/10.2519/jospt.2017.0302.

28. Zung WW. A rating instrument for anxiety disorders. Psychosomatics. 1971; 12(6):371-9. https://doi.org/10.1016/50033-3182(71)71479-0.

29. Zung WW. A SELF-RATING DEPRESSION SCALE. Archives of general psychiatry. 1965;12(1):63-70. https://doi.org/10.1001/archpsyc.1965.0172031 0065008.

30. Patel AA, Donegan D, Albert T. The 36-item short form. The Journal of the American Academy of Orthopaedic Surgeons. 2007;15(2):126-134, DOI: https://doi.org/10.5435/00124635-200702000-00007.

31. Walton DM, Macdermid JC, Nielson W, Teasell RW, Nailer T, Maheu P. A descriptive study of pressure pain threshold at 2 standardized sites in people with acute or subacute neck pain. J Orthopaedic Sports Physical Therapy. 2011;41(9):651-7. https://doi.org/10.2519/jospt.2011.3 667.

32. Klauser AS, Miyamoto H, Bellmann-Weiler R, Feuchtner GM, Wick MC, Jaschke WR. Sonoelastography: musculoskeletal applications. Radiology. 2014;272(3):622-33. https://doi.org/10.1148/radiol.14121765.

33. Wang YJ, Zhang $L$, Song K. [Verification of the theory of "Lieque (LU 7) for the disorders of the head and neck" based on infrared thermography]. Zhongguo zhen jiu = Chinese acupuncture \& moxibustion. 2019;39(2):169-72.

34. Yao XJ, Liu JW. [Observation on clinical efficacy of acute pain treated with the intervention of different time of needle retention]. Zhongguo zhen jiu = Chinese acupuncture \& moxibustion. 2013;33(11):985-8.

35. Lei Q. Zheng's Wentong acupuncture method treats 35 cases of nerve root cervical spondylopathy. Traditional Chinese Medicinal Research. 2020;33(01): 46-9.

36. Zheng $H$, Tian XP, Li Y, Liang FR, Yu SG, Liu XG, et al. Acupuncture as a treatment for functional dyspepsia: design and methods of a randomized controlled trial. Trials. 2009;10(1):75. https://doi.org/10.11 86/1745-6215-10-75

37. Zhao L, Li D, Zheng H, Chang X, Cui J, Wang R, et al. Acupuncture as adjunctive therapy for chronic stable angina: a randomized clinical trial. JAMA internal medicine. 2019;179(10):1388-97. https://doi.org/10.1001/jama internmed.2019.2407.

38. Wang VC, Mullally WJ. Pain neurology. The American journal of medicine. 2020;133(3):273-80. https://doi.org/10.1016/.amimed.2019.07.029.

39. Montoya P, Larbig W, Braun C, Preiss H, Birbaumer N. Influence of social support and emotional context on pain processing and magnetic brain responses in fibromyalgia. Arthritis and rheumatism. 2004;50(12):4035-44. https://doi.org/10.1002/art.20660.

40. Chen W, Hou X, Chen J, Zhang D, Ye G, Lin C, et al. [MRI pain matrix regional homogeneity in cervical spondylosis of neck type treated with acupuncture at multiple acupoints]. Zhongguo zhen jiu = Chinese acupuncture \& moxibustion. 2015;35(10):1005-9.

41. Beinert K, Mouthon A, Keller M, Mouthon M, Annoni JM, Taube W. Neural correlates of maladaptive pain behavior in chronic neck pain - a single case control fMRI study. Pain physician. 2017;20(1):E115-e25.

42. Bernabéu-Sanz Á, Mollá-Torró JV, López-Celada S, Moreno López P, Fernández-Jover E. MRI evidence of brain atrophy, white matter damage, and functional adaptive changes in patients with cervical spondylosis and prolonged spinal cord compression. European radiology. 2020;30(1):357-69. https://doi.org/10.1007/s00330-019-06352-z.

43. Zhang Y, Zhang H, Nierhaus T, Pach D, Witt CM, Yi M. Default mode network as a neural substrate of acupuncture: evidence, challenges and strategy. Frontiers in neuroscience. 2019;13:100. https://doi.org/10.3389/ fnins.2019.00100.

44. Yan CQ, Huo JW, Wang X, Zhou P, Zhang YN, Li JL, et al. Different degree centrality changes in the brain after acupuncture on contralateral or ipsilateral acupoint in patients with chronic shoulder pain: a resting-state fMRI study. Neural plasticity. 2020;2020:5701042.

45. Mawla I, Ichesco E, Zöllner HJ, Edden RAE, Chenevert T, Buchtel H, et al. Greater somatosensory afference with acupuncture increases primary somatosensory connectivity and alleviates fibromyalgia pain via insular GABA: a randomized neuroimaging trial. Arthritis \& rheumatology (Hoboken, NJ). 2020

46. Makary MM, Lee J, Lee E, Eun S, Kim J, Jahng GH, et al. Phantom acupuncture induces placebo credibility and vicarious sensations: a parallel 
fMRI study of low back pain patients. Scientific reports. 2018;8(1):930. https://doi.org/10.1038/s41598-017-18870-1.

47. Yu SW. Lin SH, Tsai CC, Chaudhuri KR, Huang YC, Chen YS, et al.

Acupuncture effect and mechanism for treating pain in patients with

Parkinson's disease. Frontiers in neurology. 2019;10:1114.

\section{Publisher's Note}

Springer Nature remains neutral with regard to jurisdictional claims in published maps and institutional affiliations.

Ready to submit your research? Choose BMC and benefit from:

- fast, convenient online submission

- thorough peer review by experienced researchers in your field

- rapid publication on acceptance

- support for research data, including large and complex data types

- gold Open Access which fosters wider collaboration and increased citations

- maximum visibility for your research: over $100 \mathrm{M}$ website views per year

At $\mathrm{BMC}$, research is always in progress.

Learn more biomedcentral.com/submissions 